\section{ORIENTACIÓN CULTURAL HACIA LA INNOVACIÓN EN EMPRESAS. Un enfoque sociológico del Sistema de Innovación de Galicia}

\author{
Universidad de Vigo, España. \\ cjardon@uvigo.es \\ Miguel GonZÁLEZ-LoUREIRO
Universidad de Vigo, España.
mloureiro@uvigo.es \\ José Pita-CAstelo \\ Universidad de Vigo, España. \\ jpita@uvigo.es
}

Carlos M. Fernández-Jardón Fernández

Cómo citar este artículo / Citation: Fernández-Jardón Fernández, C. M.; M. González-Loureiro y J. Pita-Castelo (2016). "Orientación cultural hacia la innovación en empresas. Un enfoque sociológico del Sistema de Innovación de Galicia", Revista Internacional de Sociología, 74 (2): e035. Doi: http://dx.doi.org/10.3989/ris.2016.74.2.035

\begin{abstract}
Resumen
Las características sociológicas de las organizaciones influyen en los resultados del proceso de innovación. Por tanto, es necesario conocer cuáles de esas características describen mejor a las empresas con mayores resultados de innovación, entre las que se encuentra la cultura de innovación existente en un contexto dado. Este estudio analiza dichos elementos sociológicos en una muestra de 441 empresas del Sistema Regional de Innovación de Galicia. Mediante métodos multivariantes se detectaron dos perfiles empresariales de orientación cultural hacia la innovación: un $47 \%$ con una clara orientación hacia la innovación y un 53 \% con una orientación menor. Los resultados evidenciaron la existencia de una asociación positiva entre la orientación cultural hacia la innovación y el desempeño innovador. Nuestra evidencia señala que la cultura de innovación es una dualidad capacidad-actitud, potencialidad-acción. Esto abre nuevas perspectivas de investigación a la sociología de la innovación en los sistemas regionales de innovación.
\end{abstract}

\section{Palabras Clave}

Cultura de innovación; Desempeño organizativo; Orientación hacia la innovación; Resultados de innovación; Sociología de la Innovación.

\section{CULTURAL ORIENTATION IN FIRMS TO INNOVATION. A sociological approach to the Galician Innovation System}

Copyright: (C) 2016 CSIC. Este artículo de acceso abierto distribuido bajo los términos de la licencia Creative Commons Attribution-Non Commercial (by-cn) Spain 3.0.

Recibido: 14/04/2014. Aceptado: 15/05/2015. Publicado on line: 19/05/2016

\section{Abstract}

The organizations' sociological characteristics have an impact on the performance of the innovation process. Therefore, knowing the sociological characteristics that best describe those ventures holding higher innovation results is particular relevant. Among these features, the culture of innovation in a certain context has been highlighted as the most relevant. This study aims at analyzing the elements shaping the cultural orientation to innovate on a sample of 441 firms within the Galician Regional System of Innovation. By means of multivariate methods, two types of cultural orientation to innovate were detected among ventures: a $47 \%$ of the sample held a clear orientation to innovate, while a $53 \%$ held a lesser orientation. The results showed the existence of a positive association between the cultural orientation to innovate and the innovative performance. Our evidence points out the culture of innovation as the duality capacity-attitude, i. e. potentiality-action. This opens new research avenues for delving deeper into the innovation sociology within the Regional Systems of Innovation.

\section{KEYWORDS}

Culture; Innovation Performance; Innovation Orientation; Organizational Performance; Sociology of Innovation. 


\section{INTRODUCCIÓN}

La sociología de la innovación es un campo de investigación multidisciplinar dentro de las ciencias sociales, habitualmente considerada un híbrido emergente desde la sociología de la economía y de la ciencia (Fernández Esquinas 2012). En una revisión de este campo como futuro ámbito de investigación con corpus propio, Fernández Esquinas (2012) sugiere la visión de la innovación como un proceso eminentemente social y grupal. Dicho enfoque sociológico también se encuentra implícito en enfoques complementarios, ya que la innovación es un proceso psicológico que facilita la transformación de roles de trabajo de individuos, de equipos y de organizaciones en los deseados estados futuros (Rank et al. 2004). Esta perspectiva psicológica llevada a un nivel de análisis meso nos llevaría al estudio de la sociología de la innovación como un proceso de un grupo de individuos organizados de una determinada manera, influidos por el entorno en el que se localiza la unidad de análisis e influyendo sobre dicho entorno. O dicho de otra manera, la innovación es un fenómeno colectivo desarrollado en sociedad por las personas imbuidas en un contexto históricocultural concreto (Granovetter 1985).

Dentro del estudio de dicho proceso de innovación, sus antecedentes y consecuentes han sido estudiados desde diversos enfoques. Así, los enfoques psicosociológicos enfatizan la necesidad de analizar aspectos determinantes del comportamiento individual y de las organizaciones, mientras que parecen en ocasiones contrapuestos a las recomendaciones desde enfoques económicos para la gestión de la innovación. Estos señalarían que se trataría de un problema de maximización, de invertir en todos los antecedentes de resultados positivos de innovación (Stock y Zacharias 2011). Sin embargo, sabemos que las empresas tienen recursos y capacidades limitadas y no pueden invertir en absolutamente todos los facilitadores de la innovación, sino en solo los más determinantes.

Entre los antecedentes de resultados positivos de innovación suele destacarse muy frecuentemente la orientación cultural hacia la innovación o la cultura organizativa de innovación como uno de los de mayor impacto (Smith et al. 2008; Stock y Zacharias 2011). La orientación cultural hacia la innovación no es unidimensional tal como han demostrado diversos estudios como el de Dobni (2008) sobre una escala de cultura de innovación o el estudio de Wang y Ahmed (2004) sobre una escala de 20 ítems.

Por tanto, el debate debe orientarse ahora hacia la identificación de los diversos aspectos culturales de la innovación más asociados con la obtención de altos resultados de innovación. Su estudio es relevante porque la obtención de elevados resultados de innovación son indicadores de una elevada capacidad de innovación (innovativeness) en una empresa (Santos et al. 2011) y la orientación cultural a innovar influye en dicha capacidad.
Así, el estudio comparativo de las características socioculturales de las empresas con un perfil innovador respecto a las que tienen un perfil menos orientado a la innovación cobra especial relevancia. Sin embargo, poco se ha investigado hasta la fecha en la caracterización de las organizaciones innovadoras desde la perspectiva de la sociología de la innovación, tal como enfatiza Fernández Esquinas (2012). El presente trabajo contribuye a dicho ámbito mediante un estudio comparativo entre empresas más y menos orientadas culturalmente a la innovación.

Para ello, en primer lugar, analizamos el marco teórico de la conceptualización de la orientación cultural hacia la innovación. Posteriormente, exponemos la metodología seguida. Tras la discusión de resultados aplicada al caso del sistema de innovación de Galicia, presentamos las principales conclusiones y sugerimos futuras líneas de investigación en la agenda de la sociología de la innovación.

\section{Orientación cultural hacia la innovación en LA EMPRESA}

En sentido amplio, la orientación hacia la innovación, tal como documentan Siguaw et al. (2006), es un constructo amplio y multidimensional alrededor de un ethos organizativo, un carácter distintivo que impulsa a la organización a innovar de forma constante como filosofía de funcionamiento que la lleva a un proceso de obtención de innovaciones de forma continua. A la vista de la revisión de conceptualizaciones de los últimos 35 años, dichos autores proponen que la orientación hacia la innovación es una estructura de conocimiento multidimensional que guía el comportamiento de todas las partes de la organización y de sus individuos. En su propuesta, incluyen aspectos de filosofía de aprendizaje, dirección estratégica y de aclimatación transfuncional, este último en el sentido de estructura de conocimiento orientada a adaptarse a un nuevo clima organizativo que es cambiante continuamente cuando la innovación es un objetivo de la organización.

Dentro de esta conceptualización de orientación hacia la innovación, subyace un componente cultural que dichos autores no explicitan claramente, pero que permite entender la orientación hacia la innovación como una herramienta que ayuda a conseguir unos objetivos organizativos (innovar en este caso) manteniendo las partes organizativas alineadas con dichos objetivos. Así, en su estudio sobre cómo la cultura organizativa puede ser una fuente de ventajas competitivas sostenidas, Barney (1986: 657) la define como "un conjunto complejo de valores, creencias, asunciones y símbolos que definen la manera en que una empresa desarrolla su negocio". Consecuentemente, en la orientación hacia la innovación subyace un primer componente que es la cultura de innovación o la orientación cultural hacia la innovación. En Siguaw (2006), la orientación hacia la innovación 
excede el alcance del adjetivo "cultural", ya que incluyen aspectos relativos a recursos, tecnología, empleados, operaciones y mercado. La orientación cultural hacia la innovación no es unidimensional tal como han contrastado diversos estudios (p. ej. Wang y Ahmed 2004; Dobni 2008). Stock y Zacharias (2011) en su estudio de patrones de resultados derivados de la orientación hacia la innovación incluyen entre los componentes de esta a la propia cultura organizativa. Por tanto, procede revisar los posibles componentes a considerar alrededor de la orientación cultural hacia la innovación, tanto en su componente hard de bienes tangibles (maquinaria, tipo de producto...) como soft (personas, competencias...).

Manu y Sriram (1996) conceptualizan la orientación cultural hacia la innovación como una construcción de múltiples componentes. Entre ellas, señalan aspectos relativos a inputs y outputs como la introducción de nuevos productos, los gastos en I+D, o el orden de entrada de la empresa en el mercado. Sin embargo, no contemplan aspectos influyentes en el proceso social a través del cual dichos inputs se transforman en outputs, tales como los valores, creencias y actitudes de toda la organización.

Homburg et al. (2002: 96) ven la orientación hacia la innovación como una función "del número de innovaciones que una empresa ofrece, a cuántos clientes se ofrecen estas innovaciones, y con qué fuerza se enfatizan estas innovaciones". Todos ellos son resultados de innovación, más que específicamente una orientación hacia la innovación. Por lo tanto, encontramos una primera tipología de conceptualizaciones de la orientación cultural hacia la innovación medida en términos de resultados. Sin embargo, los resultados de innovación son un output del proceso que deben servir para evaluar la eficiencia y eficacia del proceso de innovación, pero que no informan sobre el cómo.

Berthon et al. (1999) definen la orientación de innovación en términos de superioridad tecnológica. Es decir, consideran que empresas innovadoras son aquellas que "dedican su energía hacia la invención y el refinado de productos superiores". Esta conceptualización consiste tanto en la apertura de la organización a la innovación como en la propia capacidad de innovación, medida esta como la obtención de productos superiores.

Amabile (1997) sostiene que los elementos más importantes de la orientación cultural hacia la innovación son la asignación de un valor cultural y una identidad propia al hecho de innovar. Sostiene que deben enfatizarse valores y actitudes como la creatividad y una orientación positiva a asumir riesgos, a asumir y tomar parte activa en el cambio, en contraposición a la orientación hacia el mantenimiento del statu quo. De esta forma, se desarrollaría un sentido de orgullo en los miembros de la organización y un entusiasmo acerca de lo que son capaces de hacer.
En conclusión, la orientación hacia la innovación es una filosofía en la que las empresas desarrollan un sistema de normas y creencias sobre el aprendizaje y los conocimientos comunes, cuyos resultados están presentes y orientan a todas las áreas funcionales hacia la innovación. Un elemento común de las definiciones anteriores es que la orientación hacia la innovación es estratégica (Amabile 1997; Manu y Sriram 1996; Siguaw et al. 2006), puesto que pretende obtener un positivo desempeño organizativo en innovación que permita a la organización situarse por encima de los competidores.

En términos de proceso, podemos considerar dicha orientación como un plan intencional, un comportamiento calculado o una intención estratégica en el ámbito del comportamiento organizativo sobre la base de la teoría del comportamiento planificado de Ajzen (1991). Dicha teoría evidenció que, como paso previo a la acción, el individuo desarrolla un conjunto de actitudes tendentes a conseguir el éxito en la acción subsiguiente, por lo que Ajzen (1991) concluía que los individuos planifican su actividad sobre un objetivo a largo plazo. Extendiendo dicha teoría al ámbito de la innovación, cabría esperar que los directivos planifiquen el comportamiento colectivo de su organización, promoviendo para ello un conjunto de actitudes favorables, una orientación cultural tendente a obtener un resultado (objetivo) que, en este caso, sería la obtención e introducción de productos o procesos innovadores. Para ello, es necesario que la colectividad (i. e., la organización) desarrolle primero una serie de capacidades que potencialmente les permitirán una puesta en acción posterior. Sin embargo, la capacidad es una condición necesaria pero no suficiente para el éxito, ya que expresa una potencialidad, un "ser capaz de hacer". Será necesario que los medios, los recursos implementados en la acción subsiguiente, sean coherentes con el objetivo planeado y las actitudes. Por consiguiente, podemos definir la orientación cultural hacia la innovación como el binomio capacidad-acción que permite la obtención de resultados positivos en el proceso de innovación de la organización.

La cultura condiciona el comportamiento de las empresas (Garmendia 2004) y de la sociedad en su conjunto. Por ese motivo, es esencial detectar los factores culturales que afectan a los resultados de la empresa. En particular, sostenemos que la empresa obtiene un mejor desempeño en su proceso innovador a partir de la orientación cultural hacia la innovación. Normalmente, la orientación hacia la innovación se inscribe en el ámbito cultural y a partir de ahí condiciona la creación de competencias distintivas (Prahalad y Hamel 1990), habitualmente orientadas hacia la innovación, que permiten al empresario obtener mejores resultados de innovación, y que, finalmente, se manifiestan en un mejor desempeño de la empresa (Siguaw et al. 2006). Por consiguiente, los 
factores culturales son esenciales para conocer cómo es el proceso innovador en la empresa en cuanto a un comportamiento organizativo planificado.

En la práctica, las mediciones de la cultura de innovación han puesto el foco de estudio en varios niveles de análisis. Así, Pérez-Díaz y Rodríguez (2010) analizan dos subpoblaciones de jóvenes entre 18-24 años y entre 25 y 29 años en su informe sobre la cultura de la innovación de los jóvenes españoles en el marco europeo. Según los propios autores, la medición realizada no es tanto la cultura de innovación, como una agregación de indicadores generales de cultura no necesariamente ligados a la innovación en las empresas, tales como indicadores de justicia, de horizonte vital, o de inteligencia, entre otros. Su unidad de análisis es, por tanto, una parte de la sociedad. Ésta es el contexto en el que nuestro objeto de estudio, la empresa, desarrolla sus innovaciones.

Los diversos institutos estadísticos oficiales también han desarrollado su propio cuestionario coordinado por el organismo europeo Eurostat. Así, en España, el INE lleva a cabo la Encuesta sobre Innovación en las Empresas, y algunos organismos autónomos como el IGE desarrollan su Encuesta de Innovación Empresarial. Entre los aspectos incluidos en dichos cuestionarios, apenas figuran explícitamente aspectos relacionados con la cultura. Esencialmente, se centran en factores productivos dedicados a la innovación en términos de recursos humanos, financieros y tecnológicos, objetivos y procesos de innovación y en los resultados de innovación obtenidos, así como en cuestiones descriptivas.

Por tanto, se observa un cierto déficit de medición de estos aspectos culturales en los cuestionarios oficiales cuya unidad de análisis es la empresa.

\section{Aspectos METODOLÓgIcos}

El proceso de análisis de la orientación cultural hacia la innovación se contrastó en empresas del Sistema de Innovación de Galicia. En este epígrafe, se detallan los aspectos metodológicos del estudio: la muestra, las variables seleccionadas y los métodos estadísticos utilizados.

\section{Población y muestra}

La población objeto de estudio fueron las empresas del Sistema de Innovación de Galicia. El contexto económico influye notablemente en la orientación innovadora, por lo que procede aportar unas breves pinceladas para el caso objeto de estudio. La economía gallega ha pasado por diferentes etapas. El período estudiado (2000-2002), supuso un fuerte crecimiento económico prolongado desde el año 1997 hasta el 2007 (Vence 2010). No obstante, las estadísticas oficiales del Instituto Nacional de Estadística o del Instituto Galego de Estatística no ofrecen datos sobre innovación en las empresas hasta el año 2005. Según dichas estadísticas, el número de empresas innovadoras en España creció un 3,5 \% entre 2005 y 2007 (de 29.766 a 30.819), mientras que en Galicia dicho crecimiento fue del $24,6 \%$ (de 1400 a 1745). Tras el inicio de la crisis en 2008 y hasta 2012, dicho número decreció un 41 \% hasta las 18.077 empresas en España, mientras que en Galicia cayó un 37,4 \% hasta las 1092. Una descripción más detallada del Sistema de Innovación de Galicia puede ser consultada en GonzálezGurriarán y Figueroa-Dorrego (2005).

Por lo tanto, el Sistema Regional de Innovación de Galicia es un espacio sociológico de innovación relevante para el estudio, ya que ha ganado en importancia y resultados, con un crecimiento en el número de empresas innovadoras mayor que la media estatal y una caída menor durante la crisis.

La muestra de empresas se seleccionó a partir de la base de datos ARDAN (Consorcio de la Zona Franca de Vigo) y la base de datos SABI. Se seleccionaron las empresas en función de la actividad, localización y el valor añadido bruto. La generación de valor nos parecía importante para estudiar la cultura de innovación, ya que uno de los objetivos de la innovación es dotar a la empresa de productos y servicios diferenciados que ayuden a generar un mayor valor añadido sobre los inputs (González-Loureiro y Figueroa-Dorrego 2012). La Clasificación Nacional de Actividades Económicas (CNAE) permitió definir un conjunto de grandes sectores de actividades empresariales bajo un elemento fundamental común (una materia prima, un producto o un servicio), siguiendo la filosofía de Marshall (1932) de distrito industrial. El diseño de la muestra se hizo mediante muestreo estratificado en función del sector de actividad económica, de manera que la muestra fuera proporcional al valor añadido bruto generado por ese conjunto de empresas. Finalmente se seleccionaron 608 empresas.

Las encuestas se llevaron a cabo entre los meses de julio y diciembre de 2005, correspondiendo los resultados de innovación a un período anterior (20002002). Primero se les envió por correo la encuesta. Con posterioridad al envío de las encuestas, desde el contacto con la empresa (recepción, responsable de cubrirla, asesoramiento, etc.) hasta la recepción de la información debidamente cumplimentada se realizó un seguimiento de su estado. Se visitó a las empresas en caso de haber sido necesario.

\section{La Tabla 1 detalla la ficha técnica de la encuesta.}

Como resultado del proceso se obtuvo una tasa de respuesta del $72,5 \%$. Se comprobó que la muestra final se ajustaba a la población de partida, mediante un test de bondad de ajuste (Newbold et al. 2002). Tal como se muestra en la Tabla 2, el ajuste del número de empresas en la muestra final sobre la realidad poblacional fue adecuado según 
dicho test, a la vista de que la obtención de elevado valor añadido bruto es uno de los objetivos del desarrollo de innovaciones González-Loureiro y Figueroa-Dorrego 2012).

Tabla 1.

Ficha técnica de la encuesta

\begin{tabular}{ll}
\hline Población total & $\mathbf{1 7 4 . 5 0 3}$ \\
\hline Tamaño muestra & 608 \\
\hline $\mathrm{N} .{ }^{\circ}$ encuestas & 441 \\
\hline Tasa de respuesta & $\mathbf{7 2 , 5 \%}$ \\
\hline Tipo de muestreo & $\begin{array}{l}\text { Estratificado con cuota mínima } \\
\text { y resto proporcional y aleatorio } \\
\text { dentro de la cuota }\end{array}$ \\
\hline Error en tablas $(\mathrm{p}=\mathrm{q})$ & $4,64 \%$ \\
\hline Nivel de confianza & $95 \%$ \\
\hline
\end{tabular}

Tabla 2.

Significación de la muestra final de empresas

\begin{tabular}{|c|c|c|c|c|}
\hline $\begin{array}{l}\text { Agrupación } \\
\text { de } \\
\text { actividades }\end{array}$ & $\begin{array}{c}\text { Códigos } \\
\text { CNAE93- } \\
\text { Rev1 } \\
\text { incluidos }\end{array}$ & $\begin{array}{c}\text { Número de } \\
\text { empresas } \\
\text { muestra } \\
\text { final }\end{array}$ & $\begin{array}{c}\% \\
\text { sobre la } \\
\text { muestra } \\
\text { final }\end{array}$ & $\begin{array}{c}\% \text { en } \\
\text { valor } \\
\text { añadido } \\
\text { bruto }\end{array}$ \\
\hline $\begin{array}{l}\text { Industria } \\
\text { alimentaria }\end{array}$ & $01 ; 05 ; 15$ & 50 & $11,3 \%$ & $10,7 \%$ \\
\hline $\begin{array}{l}\text { Industrias } \\
\text { metálicas y } \\
\text { automoción }\end{array}$ & $\begin{array}{c}11 \text { а } 13 ; 27 \\
\text { a } 29 ; 31 ; \\
34 ; 35 ; 50 ;\end{array}$ & 100 & $22,7 \%$ & $19,5 \%$ \\
\hline $\begin{array}{c}\text { Construcción } \\
\text { y } \\
\text { relacionados }\end{array}$ & $\begin{array}{c}02 ; 10 ; 14 ; \\
20 ; 21 ; 23 \\
\text { a 26; } 36 ; \\
40 ; 45 ; 70 ; \\
71\end{array}$ & 125 & $28,3 \%$ & $31,8 \%$ \\
\hline $\begin{array}{c}\text { TIC, } \\
\text { servicios y } \\
\text { comercio }\end{array}$ & $\begin{array}{c}30 ; 32 ; 33 \\
37 ; 41 ; 51 \\
52 ; 60 \text { a } \\
67 ; 72 \text { a } \\
74 ; 80 \mathrm{~B} ; \\
85 \mathrm{~B} ; 91 \\
\text { a } 93 \\
\end{array}$ & 103 & $23,4 \%$ & $23,5 \%$ \\
\hline $\begin{array}{c}\text { Turismo y } \\
\text { textil }\end{array}$ & $\begin{array}{c}17 \text { a } 19 ; \\
22 ; 55 ; 63\end{array}$ & 63 & $14,3 \%$ & $14,5 \%$ \\
\hline TOTAL & & 441 & $100,0 \%$ & $100,0 \%$ \\
\hline
\end{tabular}

\section{Metodología estadística}

La depuración de datos se hizo mediante métodos multivariantes, eliminando del análisis los valores extraños y los comportamientos conjuntos anómalos para evitar sesgar los resultados. El posterior método bietápico desarrollado es, en parte, similar al descrito por González de la Fe et al. (2012). En una primera etapa, se detectaron las variables estructurales en torno al concepto de orientación cultural hacia la innovación. En una segunda etapa, se clasificaron las empresas en dos grupos en base a dichas componentes, detectándose dos tipos de perfiles según su mayor o menor orientación cultural hacia la inno- vación. En esta segunda etapa se realizó un t-test de diferencia de medias en las variables de resultados de innovación entre ambos tipos de orientación cultural hacia la innovación.

En la primera etapa, las variables estructurales que caracterizan los aspectos genéricos de la orientación cultural hacia la innovación se construyeron mediante análisis confirmatorio de componentes principales. Se utilizó el alfa de Cronbach para evaluar la fiabilidad de los indicadores utilizados (Cronbach 1951). Dicho estadístico mide la consistencia interna del cuestionario. Se basa en el análisis de las correlaciones promedios entre los ítems referidos a un mismo aspecto, a partir de una sola administración del cuestionario. Se considera que es adecuado cuando es mayor de 0,6 (Nunnally y Bernstein 1994).

Una vez comprobada la fiabilidad de las medidas utilizadas para las variables estructurales se hizo uso del análisis exploratorio de componentes principales para detectar las dimensiones subyacentes a la orientación cultural. El análisis de componentes principales permite reducir la información que suministran diferentes variables en un número inferior de componentes que mantengan la mayoría de la información obtenida de los datos. Para evaluar la fiabilidad se hizo uso del porcentaje de varianza explicado y de la comunalidad de las variables. El primero indica la parte de información que las componentes recogen del total de información suministrada por todas las variables. Habitualmente, se exige que se sitúe en torno al $50 \%$. La comunalidad mide la parte de cada variable explicada por el modelo. Para que una variable se considere relacionada con el resto se suele exigir que dicha comunalidad sea igual o superior a 0,4 (Costello y Osborne 2005). Combinamos ambos criterios a la hora de seleccionar el número de componentes extraídas. Para interpretar las variables se utilizó una rotación Varimax (Hair et al. 2006).

En la segunda etapa, se clasificaron los grupos de empresas en base a esas dimensiones subyacentes. Dicha clasificación se llevó a cabo mediante la metodología de k-medias, que busca agrupar los sujetos observados de forma que la distancia a la media global de los grupos previamente formados sea mínima dentro del grupo y máxima entre grupos. Se probaron diferentes valores para el número de grupos y se eligió aquel que presenta más coherencia con los datos, facilitando su interpretación (Hair et al. 2006).

Finalmente, para cada uno de los grupos se comprobó su perfil respecto a los resultados de innovación. Las comparaciones de medias, realizadas mediante t-test o test no paramétricos (Kruskall Wa$\|$ lis) ${ }^{1}$, permitieron tener un contraste final sobre la fiabilidad del método para clasificar empresas más o menos orientadas culturalmente a la innovación (Newbold et al. 2002). 
El tamaño también podría influir en una mayor o menor orientación cultural hacia la innovación. Para controlarlo, caracterizamos los perfiles de la orientación cultural hacia la innovación con el tamaño de las empresas encuestadas y, siguiendo a Everitt (1977) y a Reynolds (1984), desarrollamos un análisis de asociación mediante tablas de contingencia, con la finalidad de estudiar las asociaciones entre las variables categóricas. En dichas tablas se estudia la posible asociación entre las categorías representadas en las filas y columnas comparando la distribución de las proporciones dentro de la matriz. Se utiliza el estadístico Chi-cuadrado de Pearson que contrasta la hipótesis nula de independencia entre las variables, de tal forma que para p-valores menores de 0,05 (nivel de confianza del $95 \%$ ) se rechazará esta hipótesis y, por lo tanto, se aceptará que existe alguna asociación entre las variables. En caso contrario, cualquier diferencia entre valores observados puede deberse al simple azar.

Ambas variables, orientación cultural innovadora (menos/más orientada a la innovación) y tamaño (microempresa, pequeña, mediana y grande), son ordinales por cuanto los valores que toman establecen un orden. Para estos casos, Agresti (1984) recomienda el uso de medidas de asociación simétricas tales como el estadístico Gamma, el estadístico $d$ de Somers y los estadísticos tau-b y tau-c de Kendall. El Gamma es una medida simétrica de asociación de dos variables ordinales que varía entre $-1 \mathrm{y}+1$, siendo la asociación mayor cuanto más cercana a uno en valor absoluto. El $d$ de Somers difiere del anterior en que es una medida asimétrica que incluye el número de cruces no empatados en la variable independiente. Los estadísticos tau- $b$ y tau-c de Kendall son medidas no paramétricas. El primero incluye los empates de pares, mientras que el segundo no, variando también entre $-1 \mathrm{y}+1$.

La variable tamaño ha sido categorizada siguiendo los criterios de la Recomendación de la Comisión Europea de 6-5-2003 (DOCE L124 de 20-5-2003). Así, una microempresa es aquella que tienen un balance y volumen de negocio anuales de menos de 2 millones de euros con menos de 10 trabajadores. Dichas cifras son menos de 10 millones y menos de 50 empleados para la Pyme pequeña; menos de 43 millones en balance, menos de 50 en volumen de negocio y menos de 250 trabajadores para la mediana; $y$, para la grande, todo lo que iguale o supere las cifras de corte anteriores de la mediana empresa. Los datos de la muestra según tamaño de empresa vienen recogidos en la última columna de la Tabla 6 . Siguiendo a Everitt (1977) y a Reynolds (1984), en la matriz de cruces, se compara también si la proporción de cada columna es significativamente diferente a lo que cabría esperar si siguiese una distribución aleatoria Chi-cuadrado. Dicha proporción será significativamente diferente de lo que cabría esperar si el residuo ajustado estandarizado entre valor observado y esperado es mayor que el z-valor 1,96. El signo, informará de la dirección de la diferencia. Las estimaciones fueron realizadas con el paquete estadístico SPSS (versión 15).

\section{Variables y datos}

Para evaluar la orientación cultural hacia la innovación en la unidad de análisis "empresa", se hizo uso de un cuestionario basado en escalas contrastadas en la literatura (Camisón-Zornoza et al. 2004; Wang y Ahmed 2004; Siguaw et al. 2006; Dobni 2008). En concordancia, se definieron las siguientes variables: grado de actualización tecnológica, cambio tecnológico, grado de conocimiento de innovaciones en el mercado, grado de conocimiento del uso de innovaciones por parte de competidores y cultura de innovación.

Para su medición se utilizaron una serie de cuestiones según se detalla en la Tabla 3 . Dicha tabla también recoge el alfa de Cronbach (AC) relativo a cada variable, que nos muestra que los indicadores utilizados para medirla son adecuados.

Dado que el objetivo de esta investigación es el estudio de la asociación entre cultura de innovación y resultados innovadores, solo se incluyeron variables relativas al ámbito interno de las empresas y no se incluyeron variables relativas al entorno de las empresas, aspecto que debe ser mencionado como una limitación del presente estudio. Futuras investigaciones deberían incluir dichas variables. No obstante, estudios como los de Kimberly y Evanisko (1981) o Meyer y Goes (1988) han encontrado que los factores internos organizativos tienen un mayor impacto explicativo sobre los resultados de la innovación que los factores externos. La técnica estadística empleada (análisis clúster) no busca obtener un resultado en términos de causa-efecto sino en términos de asociación entre las variables incluidas con el objetivo de categorizar empresas. Por tanto, los resultados de dicha técnica no están influidos por un posible efecto de variables omitidas (aspectos del entorno), como sí podría ocurrir en análisis de regresión.

La introducción del grado de actualización tecnológica y el cambio tecnológico como variables se justifican a partir de diversos estudios. Narver y Slater (1990) incluyen el cambio tecnológico, referido este al grado de incorporación de nuevas tecnologías, en su estudio entre la orientación cultural al mercado y el desempeño organizativo. Dicha orientación cultural al mercado fue posteriormente ligada a la orientación cultural hacia la innovación por Ahmed (1998), ya que una orientación externa al mercado y al cliente provoca una actitud cultural positiva hacia el cambio que, en definitiva, es el precursor de una orientación cultural a innovar. Caben dos hipótesis de posible vinculación con altos resultados de innovación. Por un lado, aquellas em- 
presas con carencias en el grado de actualización tecnológica haría que dichas empresas comprometiesen más recursos para innovar (Ahmed 1998), lo que estaría alineado con la idea de que el estado actual de innovación tecnológica depende de la trayectoria tecnológica seguida por la empresa (Nieto
2004). Por otro lado, asumiendo que la actualización tecnológica depende de la trayectoria seguida por la empresa, tendríamos que aquellas empresas con un elevado grado de actualización tecnológica serían las que se hubiesen orientado más hacia la innovación en etapas pretéritas.

Tabla 3.

Variables del estudio

\begin{tabular}{|c|c|c|c|c|}
\hline Variables & Escala & Ítems & Media & Desv. est. \\
\hline \multirow{11}{*}{$\begin{array}{l}\text { Grado de } \\
\text { actualización } \\
\text { tecnológica }\end{array}$} & \multirow{11}{*}{$\begin{array}{c}\text { Grado de actualización } \\
\text { tecnológica actual de su empresa } \\
\text { en las siguientes tecnologías: }\end{array}$} & - Tecnologías de la información & 3,91 & 0,851 \\
\hline & & - Tecnologías de la comunicación (TIC) & 4,03 & 0,792 \\
\hline & & - Tecnologías de automatización de la producción & 3,62 & 1,040 \\
\hline & & - Tecnologías de diseño asistido & 3,47 & 1,110 \\
\hline & & - Tecnologías de medida y ensayo & 3,43 & 1,106 \\
\hline & & $\begin{array}{l}\text { - Sistemas integrados de administración } \\
\text { (contabilidad, nóminas, etc.) }\end{array}$ & 4,00 & 0,876 \\
\hline & & $\begin{array}{l}\text { - Sistemas integrados de dirección estratégica } \\
\text { (cuadro de mando, plan estratégico, etc.) }\end{array}$ & 3,49 & 1,028 \\
\hline & & - Sistemas automatizados de gestión de inventarios & 3,57 & 1,176 \\
\hline & & - Sistemas de conexión automática con proveedores & 3,31 & 1,199 \\
\hline & & - Sistemas de conexión automática con clientes & 3,37 & 1,221 \\
\hline & & - Tecnologías de marketing & 3,31 & 1,159 \\
\hline \multirow{11}{*}{$\begin{array}{l}\text { Cambios } \\
\text { Tecnológicos } \\
\text { realizados } \\
\text { AC }=0,909\end{array}$} & \multirow{6}{*}{$\begin{array}{l}\text { Cambios tecnológicos } \\
\text { incorporados por su empresa } \\
\text { en los últimos } 2 \text { años en las } \\
\text { siguientes tecnologías: }\end{array}$} & - Tecnologías de la información & 0,42 & 0,495 \\
\hline & & - Tecnologías de la comunicación (TIC) & 0,44 & 0,497 \\
\hline & & - Tecnologías de automatización de la producción & 0,34 & 0,474 \\
\hline & & - Tecnologías de diseño asistido & 0,18 & 0,382 \\
\hline & & - Tecnologías de medida y ensayo & 0,16 & 0,368 \\
\hline & & $\begin{array}{l}\text { - Sistemas integrados de administración } \\
\text { (contabilidad, nóminas, etc.) }\end{array}$ & 0,38 & 0,485 \\
\hline & \multirow{5}{*}{$\begin{array}{c}\text { Escala Likert de } \\
1 \text { (ha realizado pocos cambios } \\
\text { tecnológicos) a } \\
5 \text { (ha realizado muchos cambios } \\
\text { tecnológicos) }\end{array}$} & $\begin{array}{l}\text { - Sistemas integrados de dirección estratégica } \\
\text { (cuadro de mando, plan estratégico, etc.) }\end{array}$ & 0,20 & 0,398 \\
\hline & & - Sistemas automatizados de gestión de inventarios & 0,28 & 0,448 \\
\hline & & - Sistemas de conexión automática con proveedores & 0,23 & 0,421 \\
\hline & & - Sistemas de conexión automática con clientes & 0,22 & 0,416 \\
\hline & & - Tecnologías & 0,16 & 0,368 \\
\hline \multirow{8}{*}{$\begin{array}{l}\text { Conocimiento } \\
\text { de } \\
\text { innovaciones } \\
\text { organizativas } \\
\text { en el mercado }\end{array}$} & \multirow{8}{*}{$\begin{array}{c}\text { Grado de conocimiento de } \\
\text { innovaciones organizativas en el } \\
\text { mercado en el que opera en los } 2 \\
\text { últimos años. } \\
\text { Escala Likert de } \\
1 \text { (conozco pocos cambios) a } \\
5 \text { (conozco muchos cambios) }\end{array}$} & - En los procesos de comercialización & 3,26 & 1,166 \\
\hline & & - En la estructura organizativa & 3,20 & 1,122 \\
\hline & & - En la cartera de productos & 3,45 & 1,187 \\
\hline & & - En los procesos productivos & 3,45 & 1,199 \\
\hline & & - En los sistemas de relación con clientes & 3,36 & 1,167 \\
\hline & & - En los mercados de compra & 3,02 & 1,151 \\
\hline & & - En los mercados de venta & 3,25 & 1,165 \\
\hline & & - En los sistemas de gestión & 3,54 & 1,053 \\
\hline \multirow{8}{*}{$\begin{array}{l}\text { Conocimiento } \\
\text { del uso de } \\
\text { innovaciones } \\
\text { organizativas } \\
\text { por parte de } \\
\text { competidores }\end{array}$} & \multirow{5}{*}{$\begin{array}{l}\text { De las innovaciones organizativas } \\
\text { en el mercado anteriores, señale } \\
\text { el conocimiento que tiene del uso } \\
\text { de innovaciones organizativas } \\
\text { implementadas por parte de } \\
\text { competidores de su empresa en } \\
\text { los últimos } 2 \text { años. }\end{array}$} & - En los procesos de comercialización & 2,58 & 0,502 \\
\hline & & - En la estructura organizativa & 2,39 & 0,511 \\
\hline & & - En la cartera de productos & 2,63 & 0,492 \\
\hline & & - En los procesos productivos & 2,59 & 0,515 \\
\hline & & - En los sistemas de relación con clientes & 2,62 & 0,487 \\
\hline & \multirow{3}{*}{$\begin{array}{c}\text { Escala Likert de } 1 \text { (sé poco de las } \\
\text { innovaciones de la competencia) } \\
\text { a } 5 \text { (sé mucho de las innovaciones } \\
\text { de la competencia) }\end{array}$} & - En los mercados de compra & 2,53 & 0,500 \\
\hline & & - En los mercados de venta & 2,62 & 0,487 \\
\hline & & - En los sistemas de gestión & 2,57 & 0,510 \\
\hline
\end{tabular}

(Cont. pág. sig.) 
Tabla 4.

Variables del estudio (cont.)

\begin{tabular}{|c|c|c|c|c|}
\hline Variables & Escala & Items & Media & $\begin{array}{l}\text { Desv. } \\
\text { Est. }\end{array}$ \\
\hline $\begin{array}{l}\text { Grado de satisfacción } \\
\text { con los resultados de } \\
\text { innovación }\end{array}$ & \multicolumn{2}{|c|}{$\begin{array}{l}\text { Grado de satisfacción con los resultados de innovación obtenidos en su empresa } \\
\text { en los últimos } 3 \text { años } \\
\text { - } \quad \text { Escala de Likert de } 1 \text { (nada satisfecho) a } 5 \text { (muy satisfecho) }\end{array}$} & 3,52 & 0,894 \\
\hline \multirow{6}{*}{$\begin{array}{l}\text { Existencia de } \\
\text { departamento } \\
\text { orientado a la } \\
\text { innovación }\end{array}$} & \multirow{6}{*}{$\begin{array}{l}\text { Escala ponderada en función } \\
\text { de la relación del departamento } \\
\text { existente con la innovación } \\
\quad(\text { escala de } 1 \text { a } 5)\end{array}$} & - $\quad$ Innovación (5) & $0,10\left(^{*}\right)$ & 0,306 \\
\hline & & - $\quad \mathrm{I}+\mathrm{D}(5)$ & $0,21\left(^{*}\right)$ & 0,405 \\
\hline & & - $\quad$ Diseño (4) & $0,17\left(^{*}\right)$ & 0,372 \\
\hline & & - $\quad$ Calidad (3) & $0,37\left(^{*}\right)$ & 0,483 \\
\hline & & - $\quad$ Métodos y procedimientos (2) & $0,15\left(^{*}\right)$ & 0,362 \\
\hline & & - $\quad$ Gestión medioambiental y Otros (1) & $0,21\left(^{*}\right)$ & 0,376 \\
\hline \multirow[t]{7}{*}{ Cultura de innovación: } & \multirow{14}{*}{$\begin{array}{c}\text { Escala Likert de } 1 \text { (nada o poco } \\
\text { de acuerdo) a } \\
5 \text { (muy de acuerdo) }\end{array}$} & $\begin{array}{l}\text { - Existe una actitud constante por incrementar } \\
\text { las inversiones en nuevas tecnologías }\end{array}$ & 3,51 & 1,037 \\
\hline & & $\begin{array}{l}\text { - Su empresa busca constantemente información } \\
\text { sobre las nuevas tecnologías que pueda aplicar }\end{array}$ & 3,63 & 1,022 \\
\hline & & $\begin{array}{l}\text { - La innovación forma parte de la estrategia } \\
\text { básica de la empresa }\end{array}$ & 3,40 & 1,201 \\
\hline & & $\begin{array}{l}\text { - La inversión en innovación seguro que genera } \\
\text { mayor rentabilidad futura }\end{array}$ & 3,54 & 0,987 \\
\hline & & $\begin{array}{l}\text { - En el futuro próximo, la cooperación para } \\
\text { innovar será un factor estratégico }\end{array}$ & 3,38 & 1,099 \\
\hline & & $\begin{array}{l}\text { - Existe una preocupación constante por detectar } \\
\text { las innovaciones en la competencia }\end{array}$ & 3,62 & 1,016 \\
\hline & & $\begin{array}{l}\text { - La dirección general se preocupa por seguir } \\
\text { directamente los temas de innovación de la } \\
\text { empresa }\end{array}$ & 4,00 & 0,972 \\
\hline \multirow{7}{*}{$\begin{array}{l}\text { Actitud } \\
\text { cultural de la } \\
\text { organización } \\
\text { hacia la } \\
\text { innovación } \\
\text { AC }=0,822\end{array}$} & & $\begin{array}{l}\text { - Se incorporan habitualmente mejoras en los } \\
\text { productos, procesos y sistemas }\end{array}$ & 3,66 & 0,973 \\
\hline & & $\begin{array}{l}\text { - La empresa se adapta a los cambios del } \\
\text { entorno rápida y adecuadamente }\end{array}$ & 3,47 & 0,890 \\
\hline & & $\begin{array}{l}\text { - La dirección de la empresa se preocupa de que } \\
\text { los técnicos de la empresa tengan actualizados } \\
\text { sus conocimientos }\end{array}$ & 3,69 & 0,992 \\
\hline & & $\begin{array}{l}\text { - En todos los ámbitos de la empresa existe } \\
\text { una preocupación continua por la calidad del } \\
\text { producto o servicio }\end{array}$ & 4,16 & 0,805 \\
\hline & & - Se potencia la creatividad de los trabajadores & 3,38 & 1,048 \\
\hline & & $\begin{array}{l}\text { - Existe una cultura de acumular y transmitir las } \\
\text { experiencias a los nuevos trabajadores }\end{array}$ & 3,82 & ,820 \\
\hline & & $\begin{array}{l}\text { - Existe un inventario actualizado de la dotación } \\
\text { tecnológica de la empresa }\end{array}$ & 3,51 & 1,158 \\
\hline
\end{tabular}

$\left({ }^{*}\right)$ media de la escala dicotómica original $(0=$ no existe; $1=$ existe $)$.

Por tanto, el grado de actualización tecnológica es un elemento a tener cuenta, así como el cambio tecnológico. Así, Wang y Ahmed (2004), incluyen diversos ítems relacionados en su medición del constructo de innovativeness. Dobni (2008) lo denomina infraestructura para la innovación en el sentido de la tecnología disponible en la empresa.

Respecto al grado de conocimiento de innovaciones en el mercado y al grado de conocimiento del uso de innovaciones por parte de competidores, en su modelo marco para investigar la orientación cultural hacia la innovación, Siguaw et al. (2006) sugieren que el grado en el que la empresa tiene conocimiento de las innovaciones existentes en un mercado dado forma parte de la orientación al mercado. Kimberly y Evanisko (1981) encuentran que las empresas altamente orientadas al exterior tienen más mecanismos para obtener información relevante que les permite desarrollar sus innovaciones. La orientación al exterior es una de las dimensiones utilizadas por Cameron y Quinn (1999) para definir sus tipologías de cultura organizativa, junto a la dimensión que confronta estabilidad/control frente a flexibilidad/discrecionalidad. Sobre dicha base, Naranjo-Valencia et al. (2010) encuentran que una mayor apertura al exterior está asociada a mayores innovaciones. Por tanto, en un comportamiento planificado, la organización primero trata de captar infor- 
mación sobre las posibilidades actuales y las trayectorias tecnológicas seguidas por otros, para luego dar los pasos necesarios para desarrollar sus innovaciones.

Finalmente, se ha demostrado que el clima organizativo tiene una gran influencia en los resultados empresariales (Pritchard y Karasick 1973). Según Nerkar et al. (1996), la satisfacción incide en tres planos respecto al desempeño de los equipos de innovación. Primero, la satisfacción social, que incide en la interacción de los individuos dentro del grupo de trabajo. Segundo, la satisfacción instrumental, que se relaciona con la manera en que progresa la tarea a realizar. Y tercero, la satisfacción egocéntrica, centrada en cómo los resultados satisfacen las aspiraciones individuales de los miembros del equipo. Por tanto, se espera que una mayor satisfacción con los resultados de innovación obtenidos influyan en una mayor motivación para continuar innovando, lo que facilitaría una mayor orientación cultural hacia la innovación finalmente. Por ello, hemos medido el nivel organizativo mediante una pregunta directa al directivo responsable de la empresa, puesto que es el directivo encargado de decidir en qué medida se refuerza la orientación cultural hacia la innovación. Como variable que permita controlar el nivel de la sub-unidad organizativa, se incluyó una pregunta directa sobre si existía una unidad organizativa cuyos objetivos y actividad fuese específicamente la innovación, ya que la existencia de una unidad organizativa orientada específicamente a desarrollar las innovaciones informa sobre la orientación de la cultura organizativa, tal como señala Teece (1992).

\section{Variables de resultados de innovación}

La medición de resultados de innovación se basa primero en una conceptualización de qué se considera innovación. Así, según el Manual de Oslo (Organización para la Cooperación y Desarrollo Económico 2005), la "innovación" es la 'introducción de un producto (bien o servicio) o de un proceso, nuevo o significativamente mejorado, o la introducción de un método de comercialización o de organización nuevo aplicado a las prácticas de negocio, a la organización del trabajo o a las relaciones externas'. Para que exista innovación, por tanto, son necesarios dos aspectos: en primer lugar, que exista una actividad investigadora; en segundo lugar, que el elemento innovador introducido tenga éxito en el mercado. Esto implica que primero debe existir la capacidad de innovar y, posteriormente, que debe existir un comportamiento o acción que lleve dicha potencialidad a la realidad. Ante dicha definición, se espera que se genere un elevado número de innovaciones en una sociedad en la que existen actitudes culturales positivas hacia la innovación.

Para poder valorar el poder discriminante de las anteriores variables, se utilizaron un conjunto de variables para evaluar los resultados reales de innovación (ver Tabla 5). Dichos valores se obtuvieron mediante una pregunta directa y se calculó el valor promedio durante los años 2000 a 2002. Las medias indican que los resultados de innovación han sido más bien escasos.

Tabla 5.

Variables de resultados de innovación

\begin{tabular}{|c|c|c|c|}
\hline Variables & Ítems & $\begin{array}{l}\text { Media } \\
\text { Global }\end{array}$ & $\begin{array}{l}\text { Desviación } \\
\text { estándar }\end{array}$ \\
\hline Nuevos productos de éxito & $\begin{array}{l}\text { Número de nuevos productos introducidos con éxito por término } \\
\text { medio entre } 2000 \text { y } 2002\end{array}$ & 1,933 & 3,354 \\
\hline Nuevos procesos de éxito & $\begin{array}{l}\text { Número de nuevos procesos implementados con éxito por } \\
\text { término medio entre } 2000 \text { y } 2002\end{array}$ & 0,905 & 2,709 \\
\hline $\begin{array}{l}\text { Cuota de mercado de nuevos } \\
\text { productos }\end{array}$ & $\begin{array}{l}\text { Cuota de mercado por término medio entre } 2000 \text { y } 2002 \text { de los } \\
\text { nuevos productos }\end{array}$ & 2,891 & 10,416 \\
\hline Mejoras de productos existentes & $\begin{array}{l}\text { Número de productos mejorados por término medio entre } 2000 \\
\text { y } 2002\end{array}$ & 2,311 & 9,563 \\
\hline Mejoras de procesos existentes & $\begin{array}{l}\text { Número de procesos mejorados por término medio entre } 2000 \\
\text { y } 2002\end{array}$ & 1,737 & 4,777 \\
\hline $\begin{array}{l}\text { Tasa de renovación de productos } \\
\text { (nuevos o mejorados) }\end{array}$ & $\begin{array}{l}\text { Por término medio entre } 2000 \text { y } 2002 \text {, número de productos } \\
\text { introducidos (nuevos o mejorados) respecto a productos } \\
\text { existentes }\end{array}$ & 2,130 & 7,924 \\
\hline $\begin{array}{l}\text { Tasa de renovación de procesos } \\
\text { (nuevos o mejorados) }\end{array}$ & $\begin{array}{l}\text { Por término medio entre } 2000 \text { y } 2002 \text {, número de procesos } \\
\text { introducidos (nuevos o mejorados) respecto a procesos existentes }\end{array}$ & 1,699 & 6,082 \\
\hline Patentes y modelos de utilidad & $\begin{array}{l}\text { Número medio de patentes y modelos de utilidad nuevos entre } \\
2000 \text { y } 2002\end{array}$ & 0,346 & 1,803 \\
\hline Número de marcas registradas & Número medio de nuevas marcas registradas entre 2000 y 2002 & 0,377 & 1,098 \\
\hline Número de licencias & Número medio de nuevas licencias entre 2000 y 2002 & 0,174 & 1,087 \\
\hline $\begin{array}{l}\text { Volumen de negocio debido a las } \\
\text { innovaciones respecto al total de ventas }\end{array}$ & $\begin{array}{l}\text { Porcentaje medio de facturación respecto al total de facturación que } \\
\text { supusieron las innovaciones entre } 2000 \text { y } 2002\end{array}$ & 3,901 & 8,552 \\
\hline
\end{tabular}




\section{Discusión DE RESULTADOS: LA ORIENTACIÓN CUL- TURAL HACIA LA INNOVACIÓN EN LAS EMPRESAS DEL SISTEMA REGIONAL DE INNOVACIÓN DE GALICIA}

En primer lugar se comprobó mediante un análisis factorial confirmatorio que los ítems que componían cada una de las variables estructurales estaban estadísticamente bien definidos.

El siguiente paso fue la obtención de las variables estructurales que caracterizan la orientación cultural hacia la innovación, es decir, aquellas que resumen el conjunto de la información de todas las dimensiones de la innovación consideradas. Así, realizamos un nuevo análisis de componentes principales con todos los factores definidos previamente e incluyendo las dos variables que medían la satisfacción con los resultados de innovación y la existencia de un departamento orientado específicamente a la innovación.

Inicialmente se evaluó si las componentes utilizadas recogían suficiente información de las variables previamente definidas. Se eligieron dos componentes de acuerdo a los criterios de Costello y Osborne (2005) que explican el $54,45 \%$ de la variación total.

Para facilitar la interpretación, se hizo una rotación Varimax. La matriz de correlaciones entre las variables y los componentes una vez rotados se recoge en la Tabla 6. Se han marcado aquellas variables que están más asociadas a cada componente. La primera está relacionada fundamentalmente con variables de conocimiento y capacidades relativas a la innovación, por lo que la denominaremos capacidad organizativa de innovación. La segunda está asociada a la actitud hacia la innovación por parte de la empresa, tanto en su aspecto estratégico como cultural. Ambas, de forma conjunta componen la orien- tación cultural hacia la innovación. Estos resultados son congruentes con otros estudios que caracterizan sociológicamente el perfil cultural de las empresas (González de la Fe et al. 2012).

La primera componente sería equivalente a la dimensión de recursos y capacidades para innovar de González de la Fe et al. (2012). Las variables aquí incluidas pueden entenderse como proxies de la capacidad que tiene la empresa para absorber nuevo conocimiento externo a la organización. Así lo atestigua el hecho de incluirse variables como el conocimiento concreto de innovaciones en su mercado, el conocimiento de su uso por parte de otras empresas. Otras variables de esta dimensión recogen el comportamiento organizativo (acción) concreto ante dicho conocimiento. Así lo evidencian las variables cambio tecnológico y grado de actualización tecnológica. Es decir, ante el conocimiento de que existen innovaciones de potencial uso (variables anteriores), estas dos variables recogerían el acto de su aplicación (cambio tecnológico) y la orientación a mantenerse tecnológicamente actualizado (grado de actualización). Esta categorización implica la existencia de un código de conducta o norma, que es la de desarrollar una permanente y sistemática vigilancia tecnológica (alerta ante innovaciones) que permite a la empresa afrontar el cambio tecnológico de forma proactiva y planificada.

La segunda componente que emerge de nuestro estudio guarda relación con la actitud pro innovación definida por González de la Fe et al. (ibid.). En nuestro caso, dicha dimensión no se expresa en términos de necesidad, sino en términos positivistas del grado de orientación actual de los directivos a promover y desarrollar una orientación cultural positiva hacia un comportamiento innovador en toda la organización.

Tabla 6.

Matriz de componentes rotados de la Cultura de Innovación

\begin{tabular}{|l|c|c|}
\hline \multirow{2}{*}{ Factores } & \multicolumn{2}{|c|}{ Componente } \\
\cline { 2 - 3 } & \multicolumn{1}{|c|}{$\begin{array}{c}\text { Capacidad } \\
\text { organizativa de } \\
\text { innovación }\end{array}$} & $\begin{array}{c}\text { Actitud cultural } \\
\text { hacia la innovación }\end{array}$ \\
\hline Conocimiento del uso de las innovaciones organizativas por parte de empresas competidoras & 0,827 & 0,056 \\
\hline Cambio tecnológico & 0,768 & 0,189 \\
\hline Conocimiento de las innovaciones organizativas en el mercado & 0,720 & 0,244 \\
\hline Grado actualización tecnología & 0,563 & 0,405 \\
\hline Actitud estratégica de innovación & 0,288 & 0,799 \\
\hline Actitud cultural organizativa hacia la innovación & 0,301 & 0,746 \\
\hline Grado de satisfacción con la innovación & 0,102 & 0,577 \\
\hline Existencia de departamento orientado específicamente a la innovación & 0,076 & 0,523 \\
\hline
\end{tabular}

Método de rotación: normalización Varimax con Kaiser.

Varianza total explicada: $54,45 \%$. 
Este resultado sugiere una primera conclusión, válida en el Sistema de Innovación de Galicia, que coincide con resultados clásicos del desarrollo de competencias (Spencer y Spencer 1993). Esto es, la orientación hacia la innovación en las organizaciones empresariales está compuesta esencialmente de dos elementos: una actitud cultural hacia la innovación y unas capacidades de innovación de la organización. Es decir, primero se debe querer (actitud) y luego se debe saber hacer (capacidades). Por tanto, nuestro estudio evidencia que la orientación cultural hacia la innovación en las empresas del Sistema de Innovación de Galicia es bidimensional representado sociológicamente por la dualidad capacidad-actitud / potencialidad-acción. Estos resultados evidencian que unas empresas parecen seguir un comportamiento planificado respecto a su orientación innovadora, dotándose primero de una cierta combinación de capacidades y actitudes.

Discutidos ambos componentes, en el siguiente epígrafe se evalúa qué valores toman ambos en empresas más y menos innovadoras, así como su capacidad para discriminar entre ambos tipos de empresas en base a sus resultados reales de innovación.

\section{Clasificación de empresas en función de su orientación cultural a innovar}

A partir de las dos dimensiones estructurales de la orientación cultural hacia la innovación definidas anteriormente, se realiza la clasificación las diferentes empresas encuestadas. Se utilizó la técnica de $\mathrm{K}$-medias partiendo de las dos dimensiones obtenidas como factores. Después de diferentes ensayos con 2, 3, 4 y 5 grupos, optamos por elegir dos grupos que muestran las mayores diferencias significativas entre ellas en las variables de partida. Esta será la clasificación utilizada para realizar los perfiles y analizar las diferencias entre ambos grupos en términos de resultados de innovación.

Los valores promedios obtenidos en los dos grupos finales vienen recogidos en la Figura 1 ya estandarizados, por lo que un valor negativo indicaría una baja actitud relativa en esa variable y uno positivo, alta.

Se observa que el primer grupo tiene una cultura de innovación negativa relativamente en ambas dimensiones, mientras que el segundo grupo muestra una positiva cultura de innovación en ambas dimensiones. El primer grupo contiene 233 empresas, mientras que el segundo lo forman 208 de las 441 que forman la muestra.

Dado que los aspectos analizados hacen relación a la cultura de innovación, se denominarán a estos grupos empresas menos orientadas a la innovación en el primer caso y empresas más orientadas a la innovación en el segundo.
Figura 1

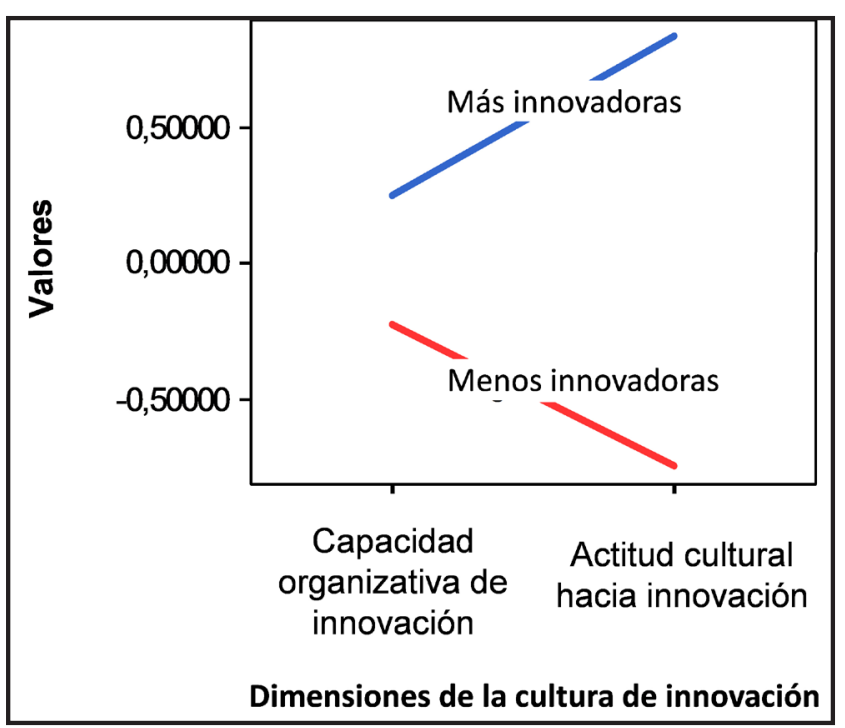

\section{Perfiles de orientación cultural hacia la innovación}

Para caracterizar cada uno de los grupos de orientación hacia la innovación y paralelamente comprobar la capacidad discriminante de ambas dimensiones y de la clasificación realizada anteriormente, se compararon los resultados medios de ambas subpoblaciones en el conjunto de variables de relativas al tamaño de las empresas y sus resultados de innovación mediante t-test.

La Figura 2 muestra las medias para cada subpoblación en las variables de resultados de innovación. Claramente, las empresas más orientadas a la innovación obtienen unos resultados superiores a las menos orientadas a la innovación, a excepción del número de marcas registradas y del número de licencias. Sin embargo, en dichos casos no se pudo realizar un contraste de medias por el bajo número de empresas con respuestas en esas variables. Por la misma causa, tampoco pudo realizarse dicho contraste en el caso del número de nuevos productos o procesos de éxitos, o en el número de patentes y modelos de utilidad, aunque los valores medios de las empresas más innovadoras estaban ligeramente por encima de los obtenidos por las menos innovadoras.

De forma significativa se observa la relevante diferencia en el porcentaje de volumen de negocio derivado de las innovaciones desarrolladas. Las empresas más innovadoras obtenían una media de un 7,3\% de ventas derivadas de sus innovaciones, mientras las menos innovadoras apenas un $3 \%$ entre los años 2000 y 2002. También se destaca la diferencia entre ambos tipos de empresa en las variables número de mejoras de productos $(6,1$ frente a 1,1$)$ y procesos $(2,9$ frente a 0,8$)$ existentes, así como en la cuota de mercado de nuevos productos (5,2 \% frente a $1,3 \%)$. Adicionalmente, las empresas más innovadoras también mantuvieron una tasa de renovación de productos $(3,7 \%$ frente a $1,0 \%)$ y procesos ( $2,8 \%$ frente a $0,9 \%$ ) superior a las menos innovadoras. 
Figura 2

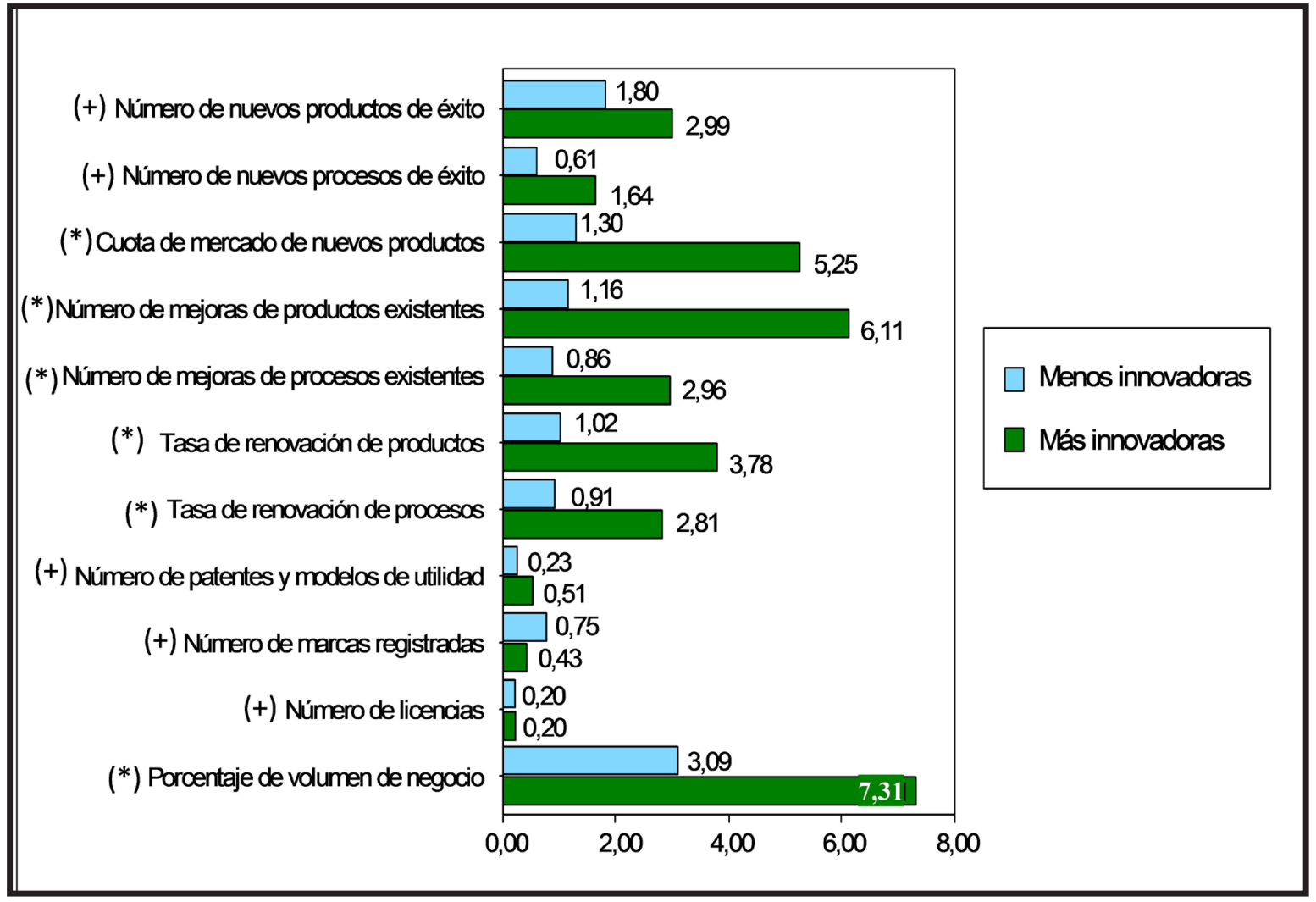

La baja respuesta en algunas de estas variables ha supuesto una limitación destacable en esta parte final del estudio. Esta baja respuesta puede deberse a que realmente las empresas encuestadas tuviesen muy bajos resultados en algunas de esas variables, tales como el número medio de licencias, el número medio de marcas registradas o el número medio de patentes y modelos de utilidad en el período de análisis considerado. De acuerdo con González de la Fe et al. (2012), es muy posible que la limitación de recursos y capacidades de las empresas condicione el registro y protección de licencias y patentes. La escasez de datos en esos aspectos, limitan en parte esta investigación, lo que debe tenerse en cuenta para futuros desarrollos.

Estos resultados evidencian que el esquema de clasificación utilizado es válido en términos discriminantes, puesto que en la mayoría de variables con suficientes casos, las empresas categorizadas como más orientadas a la innovación obtuvieron mejores resultados de sus procesos innovadores que las clasificadas como menos orientadas a la innovación. En definitiva, una mayor orientación cultural hacia la innovación, medida como una dualidad capacidad-acción, está positivamente asociada con mayores resultados de innovación.

Para evaluar la asociación entre tamaño y orientación hacia la innovación hacemos uso de una tabla de contingencia (ver Tabla 7). Primero, se observa que existe una cierta asociación entre filas y columnas (Chi-cuadrado de Pearson significativo). Dicha asociación es débil por cuanto el valor de los estadísticos apenas está cercano al límite de 0,30, excepto el Gamma que alcanza 0,31, todos ellos significativos estadísticamente. No obstante, el Gamma es el más optimista de todas las medidas de asociación. Por consiguiente, puede decirse que existe una débil aunque significativa asociación positiva entre una mayor orientación cultural hacia la innovación y un mayor tamaño de empresa. A la vista de las proporciones, se observa que, entre las microempresas, hay una significativa menor proporción de empresas más orientadas a la innovación de lo que cabría esperar, mientras que en el caso de las grandes empresas es al revés. En cambio, en los tamaños intermedios de pequeña empresa y mediana empresa la evidencia señala que las diferencias se deben al azar. Este resultado está en línea con la principal conclusión del meta-análisis llevado a cabo por Camisón-Zornoza et al. (2004): existe una débil asociación positiva entre tamaño y resultados de innovación. De hecho, al realizar los t-test de diferencia de medias entre las microempresas y las grandes empresas en las variables de resultados de innovación, la diferencia siempre fue a favor de la empresa grande pero solo en un número limitado de indicadores. Así, las empresas grandes alcanzaron significativamente mayores ratios que las microempresas en nuevos procesos de éxito, en la cuota de mercado de nuevos productos, en el número de mejoras de productos y de procesos existentes y en el número de marcas registradas, pero no en todos los demás indicadores. Por tanto, la variable relativa a la orientación hacia la innovación tiene mayor poder discriminante que el tamaño empresarial. 
Tabla 7.

Asociación entre tamaño y orientación cultural hacia la innovación de la empresa

\begin{tabular}{|c|c|c|c|c|}
\hline \multirow{2}{*}{ Tamaño } & & \multicolumn{2}{|c|}{ Orientación cultural hacia la innovación } & \multirow{2}{*}{ Total } \\
\hline & & Menos orientada & Más orientada & \\
\hline \multirow{4}{*}{ Microempresa } & Recuento & $39 a$ & $16_{b}$ & 55 \\
\hline & Rec. Esperado & 29,1 & 25,9 & 55,0 \\
\hline & $\%$ columna & $70,9 \%$ & $29,1 \%$ & $100,0 \%$ \\
\hline & Residuo corregido estandarizado & 2,9 & $-2,9$ & \\
\hline \multirow{4}{*}{ Pequeña empresa } & Recuento & $88_{a}$ & $63_{a}$ & 151 \\
\hline & Rec. Esperado & 79,8 & 71,2 & 151,0 \\
\hline & $\%$ columna & $58,3 \%$ & $41,7 \%$ & $100,0 \%$ \\
\hline & Residuo corregido estandarizado & 1,7 & $-1,7$ & \\
\hline \multirow{4}{*}{ Mediana empresa } & Recuento & $79_{a}$ & $80_{a}$ & 159 \\
\hline & Rec. Esperado & 84,0 & 75,0 & 159,0 \\
\hline & $\%$ columna & $49,7 \%$ & $50,3 \%$ & $100,0 \%$ \\
\hline & Residuo corregido estandarizado & $-1,0$ & 1,0 & \\
\hline \multirow{4}{*}{ Gran empresa } & Recuento & $27_{a}$ & $49_{b}$ & 76 \\
\hline & Rec. Esperado & 40,2 & 35,8 & 76,0 \\
\hline & $\%$ columna & $35,5 \%$ & $64,5 \%$ & $100,0 \%$ \\
\hline & Residuo corregido estandarizado & $-3,3$ & 3,3 & \\
\hline \multirow{3}{*}{ Total } & Recuento & 233 & 208 & 441 \\
\hline & Rec. Esperado & 233,0 & 208,0 & 441,0 \\
\hline & $\%$ columna & $52,8 \%$ & $47,2 \%$ & $100,0 \%$ \\
\hline \multicolumn{2}{|c|}{ Chi-cuadrado de Pearson } & & $18,775\left(^{* * *}\right)$ & \\
\hline \multicolumn{2}{|c|}{ Gamma } & & $0,311\left({ }^{* * *}\right)$ & \\
\hline \multicolumn{2}{|c|}{ d de Somers } & & $0,184\left(^{* * *}\right)$ & \\
\hline \multicolumn{2}{|c|}{ Tau-b de Kendall } & & $0,186\left(^{* \star *}\right)$ & \\
\hline \multicolumn{2}{|c|}{ Tau-c de Kendall } & & $0,221\left(^{* \star *}\right)$ & \\
\hline
\end{tabular}

Cada letra del subíndice denota un subconjunto de empresas cuyas proporciones de columna no difieren de forma significativa entre sí a un nivel de significación menor que 0,05 . Test de diferencia de proporciones con ajuste de Bonferroni. $\left({ }^{* \star *}\right)$ Valores significativos a un $p$-valor $<0,001$.

\section{Conclusiones y fUtURAS INVESTigaciones}

Este estudio ha tratado de señalar algunos aspectos sociológicos asociados a la innovación empresarial dentro de un Sistema Regional de Innovación. Para ello, se han estudiado los factores que caracterizan la orientación cultural hacia la innovación en Pymes gallegas y cómo dichos factores se asocian con un mejor rendimiento en la obtención de resultados de innovación.

La cultura de innovación en las empresas puede ser caracterizada en términos de orientación estratégica y de actitudes culturales hacia la innovación (Spencer y Spencer 1993). Además, deben incluirse también las capacidades para innovar, puesto que la cultura por sí sola no es suficiente para obtener resultados de innovación positivos. La cultura hablaría de una predisposición, que representaría el plano de la potencialidad de un comportamiento organizativo. Mientras que la capacidad indicaría el grado de compromiso real de la organización, la puesta a disposición de unos recursos para obtener un resultado positivo en su proceso innovador. Análogamente, tampoco una alta capacidad sería suficiente para caracterizar la cultura de innovación organizativa, puesto que hablaría tan solo de la dotación de recursos y capacidades organizativos, pero no informaría en absoluto sobre el ámbito de actitudes a nivel del conjunto de individuos de la organización. Esta dualidad de capacidad-actitud, de potencialidad-acción ha demostrado ser válida para discriminar aquellas empresas con un mejor desempeño de innovación.

Podría pensarse que ese proceso de innovación es fruto de una estrategia previamente definida, de un comportamiento planificado que se manifiesta en diferentes capacidades y actitudes culturales. Parece pues que la innovación de la empresa es un fruto combinado de la actitud cultural de las empresas y del microsistema de innovación que se construye internamente, especialmente relacionado con los aspectos de capacidad y actitud. 
La clasificación entre empresas más y menos orientadas a la innovación permite delimitar conclusiones en cuanto al posible efecto de la orientación cultural hacia la innovación y los resultados de innovación. Las más orientadas a la innovación tienden a ser más grandes y a tener mejores resultados de innovación.

Toda vez que hemos discriminado entre empresas más y menos orientadas culturalmente a la innovación bajo la dualidad capacidad-actitud, futuras investigaciones en el ámbito de la sociología de la innovación deberían profundizar en dicha dualidad en los otros dos subsistemas de un Sistema Regional de Innovación: los agentes del subsistema de ciencia y tecnología y los agentes del subsistema de administraciones públicas. Dicho estudio ayudaría a entender el complejo entramado de interrelaciones que conforman el Sistema Regional de Innovación. Potencialmente, la capacidad organizativa para absorber el conocimiento externo existente en los términos expresados por Cohen y Levinthal (1990) puede ser una variable muy relevante en el estudio sociológico del Sistema Regional.

Quedaría abierta la cuestión de si el Sistema Regional de Innovación tiene una influencia decisiva en la potenciación de una cultura innovadora que redunde en un incremento de los resultados de in-

\section{Notas}

[1] Los datos no verifican la normalidad, pero los t-test son válidos asintóticamente. Sin embargo, para comparar el efecto del tamaño fue necesario usar técnicas no paramétricas.

\section{Referencias Bibliográficas}

Agresti, A. 1984. Analysis of Ordinal Categorical Data. New York: John Wiley and Sons.

Ahmed, P. K. 1998. "Culture and climate for innovation". European Journal of Innovation Management 1:30-43. http://dx.doi.org/10.1108/14601069810199131

Ajzen, I. 1991. "The theory of planned behavior". Organizational Behavior and Human Decision Processes 50:179211. http://dx.doi.org/10.1016/0749-5978(91)90020-T

Amabile, T. M. 1997. "Motivating creativity in organizations: on doing what you love and loving what you do". California Management Review 40:39-58. http://dx.doi.org/10.2307/41165921

Barney, J. B. 1986. "Organizational culture: can it be a source of sustained competitive advantage?". The Academy of Management Review 11:656-665. http://dx.doi.org/10.2307/258317 / http://dx.doi.org/10.5465/AMR.1986.4306261

Berthon, P., J. M. Hulbert and L. F. Pitt. 1999. "To Serve or Create? Strategic orientations toward customers and innovation". California Management Review 42:37-58. http://dx.doi.org/10.2307/41166018

Cameron, K. S and R. E. Quinn. 1999. Diagnosing and Changing Organizational Culture: Based on the Competing Values Framework. Reading (MA): John Wiley \& Sons.

Camisón-Zornoza, C., R. Lapiedra-Alcamí, M. Segarra-Ciprés and M. Boronat-Navarro. 2004. "A Meta-analysis of Innovation and Organizational Size". Organization Studies 25:331361. http://dx.doi.org/10.1177/0170840604040039 novación de las empresas, aspecto y señalado por González de la Fe (2009). Dicha cuestión requeriría estudios comparativos y longitudinales entre empresas innovadoras de varias zonas, aspecto que queda fuera del alcance del presente estudio. No obstante, un primer resultado se vislumbra en trabajos como los de González-Loureiro y Pita-Castelo (2012) o González-Loureiro y Figueroa-Dorrego (2010; 2012). En dichos trabajos se observa que el entramado de relaciones entre entidades de I+D+i y empresas no parece tener un impacto muy elevado sobre el crecimiento acumulado en 2 y 3 años en el caso de empresas innovadoras. Por tanto, sería interesante que la agenda de investigación de la sociología de la innovación incluyese la medida de la dualidad capacidad-actitud del conjunto del Sistema Regional de Innovación, que reflejaría la orientación cultural hacia la innovación de dicho Sistema más allá de la mera consideración del agente empresa de nuestro estudio. La investigación en el campo de la sociología de la innovación debe arrojar luz sobre qué elementos sociológicos resultan determinantes en el éxito de la innovación en dos tipos diferentes de agentes que son clave para la innovación: aquellas empresas que ya cuentan con una clara orientación cultural hacia la innovación, y aquellas que cuentan con una orientación menor.
Cohen, W. M. and D. A. Levinthal. 1990. "Absorptive capacity: a new perspective on learning and innovation". Administrative Science Quarterly 35:128-152. http://dx.doi. org/10.2307/2393553

Costello, A. B. and J. W. Osborne. 2005. "Best Practices in Exploratory Factor Analysis: Four Recommendations for Getting the Most From Your Analysis". Practical Assessment, Research \& Evaluation 10:1-9.

Cronbach, L. J. 1951. "Coefficient alpha and the internal structure of tests". Psychometrika 16:297-334. http://dx.doi. org/10.1007/BF02310555

Dobni, C. B. 2008. "Measuring innovation culture in organizations: The development of a generalized innovation culture construct using exploratory factor analysis". European Journal of Innovation Management 11:539-559. http://dx.doi.org/10.1108/14601060810911156

Everitt, B. S. 1977. The Analysis of Contingency Tables. London: Chapman \& Hall.

Fernández Esquinas, M. 2012. "Hacia un programa de investigación en sociología de la innovación”. Arbor 188:5-18. http://dx.doi.org/10.3989/arbor.2012.753n1001

Garmendia, J. A. 2004. "Impacto de la cultura en los resultados de la organización". Revista Española de Investigaciones Sociológicas 108:75-96. http://dx.doi.org/10.2307/40184654

González de la Fe, T. 2009. "El modelo de Triple Hélice de relaciones universidad, industria y go- 
bierno: un análisis crítico". Arbor 185:739-755. http://dx.doi.org/10.3989/arbor.2009.738n1049

GonzálezdelaFe,T.,N.HernándezHernándezyM.VanOostrom. 2012. "Innovación, cultura y tama-o: la microempresa en una región ultraperiférica”. Arbor 188:113-134. http://dx.doi.org/10.3989/arbor.2012.753n1008

González-Gurriarán, J. y P. Figueroa-Dorrego. 2005. "Plan Estratégico de Innovación de Galicia 2010: una visión sintética". Revista madri+d 33

González-Loureiro, M. and P. Figueroa-Dorrego. 2010. "Intellectual capital on regional innovation systems: toward the momentum of growth rates of business performance". International Journal of Transitions and Innovation Systems 1:82-99. http://dx.doi.org/10.1504/ IJTIS.2010.037415

González-Loureiro, M. and P. Figueroa-Dorrego. 2012. "Intellectual capital and System of Innovation: what really matters at innovative SMEs". Intangible Capital 8:239274. http://dx.doi.org/10.3926/ic.273

González-Loureiro, M. and J. Pita-Castelo. 2012. "A model for assessing the contribution of innovative SMEs to economic growth: The intangible approach". Economics Letters 116:312-315. http://dx.doi.org/10.1016/j.econlet.2012.03.028

Granovetter, M. 1985. "Economic Action and Social Structure: The Problem of Embeddedness". The American Journal of Sociology 91:481-510. http://dx.doi.org/10.1086/228311

Hair, J., W. Black, B. Babin, R. Anderson and R. Tatham. 2006. Multivariate Data Analysis. 6th ed. Upper Saddle River: Pearson Prentice Hall.

Homburg, C., W. D. Hoyer and M. Fassnacht. 2002. "Service Orientation of a Retailer's Business Strategy: Dimensions, Antecedents, and Performance Outcomes". Journal of Marketing 66:86-101. http://dx.doi. org/10.1509/jmkg.66.4.86.18511

Kimberly, J. R. and M. J. Evanisko. 1981. "Organizational innovation: The influence of individual, organizational, and contextual factors on hospital adoption of technological and administrative innovations". Academy of Management Journal 24:689-713. http://dx.doi.org/10.2307/256170

Manu, F. A. and V. Sriram. 1996. "Innovation, marketing strategy, environment, and performance". Journal of Business Research 35:79-91. http://dx.doi.org/10.1016/0148-2963(95)00056-9

Marshall, A. 1932. Elements of Economics. Volume 1: elements of economics of industry. 3rd ed. London: Macmillan and Co., Ltd.

Meyer, A. D. and J. B. Goes. 1988. "Organizational assimilation of innovations: a multilevel contextual analysis". Academy of Management Journal 31:897-923. http://dx.doi.org/10.2307/256344

Naranjo-Valencia, J. C., R. Sanz Valle and D. Jiménez Jiménez. 2010. "Organizational culture as determinant of product innovation". European Journal of Innovation Management 13:466-480. http://dx.doi.org/10.1108/14601061011086294

Narver, J. C. and S. F. Slater. 1990. "The Effect of a Market Orientation on Business Profitability". Journal of Matketing 54:20-36. http://dx.doi.org/10.2307/1251757

Nerkar, A. A., R. G. McGrath and I. C. MacMillan. 1996. "Three facets of satisfaction and their influence on the performance of innovation teams". Journal of Business Venturing 11:167-188. http://dx.doi.org/10.1016/08839026(96)00002-X
Newbold, P., W. L. Carlson and B. M. Thorne. 2002. Statistics of Business and Economic, 5th ed. New York: Prentice Hall.

Nieto, M. 2004. "Basic propositions for the study of the technological innovation process in the firm". European Journal of Innovation Management 7:314-324. http://dx.doi.org/10.1108/14601060410565065

Nunally, J. C. and I. H. Bernstein. 1994. Psychonometric theory. 3rd ed. New York: McGraw-Hill Publishers.

Organización para la Cooperación y el Desarrollo Económicos, OCDE. 2005. Oslo manual: Guidelines for collecting and interpreting innovation data. Paris: OECD publishing.

Pérez-Díaz, V.yJ.C.Rodríguez.2010.Laculturadelainnovación de los jóvenes españoles en el marco europeo, Madrid: Fundación Cotec para la Innovación Tecnológica. PMCid:PMC2923702

Prahalad, C. K. and G. Hamel. 1990. "The Core Competence of the Corporation". Harvard Business Review 68:7991.

Pritchard, R. D. and B. W. Karasick. 1973. "The effects of organizational climate on managerial job performance and job satisfaction". Organizational Behavior and Human Performance 9:126-146. http://dx.doi.org/10.1016/0030-5073(73)90042-1

Rank, J., V. L. Pace and M. Frese. 2004. "Three avenues for future research on creativity, innovation, and initiative". Applied Psychology 53:518-528. http://dx.doi.org/10.1111/j.1464-0597.2004.00185.x

Reynolds, H. T. 1984. Analysis of Nominal Data. 2nd ed. Beverly Hills and London: Sage Publications.

Santos-Rodrigues, H., P. Figueroa Dorrego y C. Fernández Jardón. 2011. "El capital estructural y la capacidad innovadora de la empresa". Investigaciones Europeas de Dirección y Economía de la Empresa 17:69-89. http://dx.doi.org/10.1016/S1135-2523(12)60121-X

Siguaw, J. A., P. M. Simpson and C. A. Enz. 2006. "Conceptualizing Innovation Orientation: A Framework for Study and Integration of Innovation Research". Journal of Product Innovation Management 23:556-574. http:// dx.doi.org/10.1111/j.1540-5885.2006.00224.x

Smith, M., M. Busi, P. Ball and R. Van Der Meer. 2008. "Factors influencing an organisation's ability to manage innovation: a structured literature review and conceptual model". International Journal of Innovation Management 12:655-676. http://dx.doi.org/10.1142/ S1363919608002138

Spencer, L. M. y S. M. Spencer. 1993. Competencies at work. New York: Willey.

Stock, R. M. and N. A. Zacharias. 2011. "Patterns and performance outcomes of innovation orientation". Journal of the Academy of Marketing Science 39:870-888. http:// dx.doi.org/10.1007/s11747-010-0225-2

Teece, D. J. 1992. "Competition, cooperation, and innovation: Organizational arrangements for regimes of rapid technological progress". Journal of Economic Behavior \& Organization 18:1-25. http://dx.doi.org/10.1016/01672681(92)90050-L

Vence, X. 2010. "Sistema gallego de innovación y políticas de innovación: balance de una década”. Revista Galega de Economía 19:161-190.

Wang, C. L. and P. K. Ahmed. 2004. "The development and validation of the organisational innovativeness construct using confirmatory factor analysis". European Journal of Innovation Management 7:303-313. http:// dx.doi.org/10.1108/14601060410565056 


\section{Apéndice: Ítems del cuestionario}

1. En una escala de 1 (=obsoleta) a 5 (=en la última versión) señale cómo calificaría el grado de actualización de las siguientes tecnologías en su empresa.

\begin{tabular}{|l|l|l|l|l|c|}
\hline \multirow{2}{*}{$\begin{array}{l}\text { GRADO DEACTUALIZACIÓN } \\
\text { TECNOLÓGICA }\end{array}$} & \multicolumn{3}{|l}{ Obsoleto } & \multicolumn{4}{|c|}{$\begin{array}{r}\text { En la última } \\
\text { versión }\end{array}$} \\
\cline { 2 - 6 } & 1 & 2 & 3 & 4 & 5 \\
\hline Tecnologías de la información & & & & & \\
\hline $\begin{array}{l}\text { Tecnologías de la } \\
\text { comunicación }\end{array}$ & & & & & \\
\hline $\begin{array}{l}\text { Tecnologís de } \\
\text { automatización de la } \\
\text { producción }\end{array}$ & & & & & \\
\hline $\begin{array}{l}\text { Tecnologías de diseño } \\
\text { asistido }\end{array}$ & & & & & \\
\hline $\begin{array}{l}\text { Tecnologías de medida y } \\
\text { ensayo }\end{array}$ & & & & & \\
\hline $\begin{array}{l}\text { Sistemas integrados de } \\
\text { administración (contabilidad, } \\
\text { nóminas, etc.) }\end{array}$ & & & & & \\
\hline $\begin{array}{l}\text { Sistemas integrados de } \\
\text { dirección estratégica (cuadro } \\
\text { de mando, plan estratégico, } \\
\text { etc.) }\end{array}$ & & & & & \\
\hline $\begin{array}{l}\text { Sistemas automatizados de } \\
\text { gestión de inventarios }\end{array}$ & & & & & \\
\hline $\begin{array}{l}\text { Sistemas de conexión } \\
\text { automática con proveedores }\end{array}$ & & & & & \\
\hline $\begin{array}{l}\text { Sistemas de conexión } \\
\text { automática con clientes }\end{array}$ & & & & & \\
\hline Tecnologías de marketing & & & & & \\
\hline Otras (Indicar) & & & & & \\
\hline
\end{tabular}

2. En una escala de 1 (ha realizado pocos cambios) a 5 (ha realizado muchos cambios), indique el grado en que su empresa realizó innovaciones (es decir, alguna mejora respecto a las tecnologías estándar del mercado) en los últimos 2 años en alguno de los siguientes aspectos.

\begin{tabular}{|l|c|c|c|c|c|}
\hline \multirow{2}{*}{ CAMBIOS TECNOLÓGICOS } & \multicolumn{3}{|l}{$\begin{array}{l}\text { Ha realizado } \\
\text { pocos cambios }\end{array}$} & $\begin{array}{r}\text { Ha realizado } \\
\text { muchos } \\
\text { cambios }\end{array}$ \\
\cline { 2 - 6 } & 1 & 2 & 3 & 4 & 5 \\
\hline Tecnologías de la información & & & & & \\
\hline $\begin{array}{l}\text { Tecnologías de la } \\
\text { comunicación }\end{array}$ & & & & & \\
\hline $\begin{array}{l}\text { Tecnologías de automatización } \\
\text { de la producción }\end{array}$ & & & & & \\
\hline Tecnologías de diseño asistido & & & & & \\
\hline $\begin{array}{l}\text { Tecnologías de medida y } \\
\text { ensayo }\end{array}$ & & & & & \\
\hline $\begin{array}{l}\text { Sistemas integrados de } \\
\text { administración (contabilidad, } \\
\text { nóminas, etc.) }\end{array}$ & & & & & \\
\hline $\begin{array}{l}\text { Sistemas integrados de } \\
\text { dirección estratégica (cuadro de } \\
\text { mando, plan estratégico, etc.) }\end{array}$ & & & & & \\
\hline $\begin{array}{l}\text { Sistemas automatizados de } \\
\text { gestión de inventarios }\end{array}$ & & & & & \\
\hline
\end{tabular}

\begin{tabular}{|l|l|l|l|l|l|}
\hline $\begin{array}{l}\text { Sistemas de conexión } \\
\text { automática con proveedores }\end{array}$ & & & & & \\
\hline $\begin{array}{l}\text { Sistemas de conexión } \\
\text { automática con clientes }\end{array}$ & & & & & \\
\hline Tecnologías de marketing & & & & & \\
\hline Otras (Indicar) & & & & & \\
\hline
\end{tabular}

3. En una escala de 1 (=conozco pocos cambios) a 5 (=conozco muchos cambios), señale su nivel de conocimiento sobre si ha habido innovaciones importantes en los últimos dos años en los mercados de su actividad en los siguientes aspectos:

\begin{tabular}{|l|l|l|l|l|l|}
\hline \multirow{2}{*}{$\begin{array}{c}\text { CONOCIMIENTO DE INNOVACIONES } \\
\text { ORGANIZATIVAS EN EL MERCADO }\end{array}$} & \multicolumn{5}{|c|}{\begin{tabular}{l} 
En los 2 últimos años \\
\cline { 3 - 7 } \\
\cline { 4 - 7 } \\
pocos \\
cambios
\end{tabular}} \\
\hline & 1 & 2 & 3 & \multicolumn{5}{c|}{$\begin{array}{r}\text { Conozco } \\
\text { muchos } \\
\text { cambios }\end{array}$} \\
\hline En los procesos de comercialización & & & & & \\
\hline En la estructura organizativa & & & & & \\
\hline En la cartera de productos & & & & & \\
\hline En los procesos productivos & & & & & \\
\hline En los sistemas de relación con clientes & & & & & \\
\hline En los mercados de compra & & & & & \\
\hline En los mercados de venta & & & & & \\
\hline En los sistemas de gestión & & & & & \\
\hline Otras(indicar) & & & & & \\
\hline
\end{tabular}

4. En una escala de 1 (=sé poco de las innovaciones de mi competencia en ese aspecto) a 5 (sé mucho de las innovaciones de mi competencia en ese aspecto), señale su grado de conocimiento sobre las innovaciones que SU COMPETENCIA ha implantado en los últimos dos años.

\begin{tabular}{|l|l|l|l|l|l|}
\hline \multirow{2}{*}{$\begin{array}{c}\text { CONOCIMIENTO } \\
\text { DE INNOVACIONES } \\
\text { ORGANIZATIVAS } \\
\text { IMPLEMENTADAS POR SU } \\
\text { COMPETENCIA }\end{array}$} & \multicolumn{4}{|c|}{$\begin{array}{l}\text { Número de innovaciones en los } 2 \text { últimos } \\
\text { años }\end{array}$} \\
\cline { 2 - 6 } & $\begin{array}{l}\text { Sé poco de las } \\
\text { innovaciones de } \\
\text { la competencia } \\
\text { en... }\end{array}$ \\
\hline $\begin{array}{l}\text { En los procesos de } \\
\text { comercialización }\end{array}$ & & & & \multicolumn{3}{|c|}{$\begin{array}{r}\text { Sé mucho de las } \\
\text { innovaciones de } \\
\text { la competencia } \\
\text { en.. }\end{array}$} \\
\hline En la estructura organizativa & & & & & \\
\hline En la cartera de productos & & & & & \\
\hline En los procesos productivos & & & & & \\
\hline $\begin{array}{l}\text { En los sistemas de relación } \\
\text { con clientes }\end{array}$ & & & & & \\
\hline En los mercados de compra & & & & & \\
\hline En los mercados de venta & & & & & \\
\hline En los sistemas de gestión & & & & & \\
\hline Otras(indicar) & & & & & \\
\hline
\end{tabular}


5 Señale su grado de acuerdo sobre en qué medida las siguientes afirmaciones se cumplen en su empresa, en una escala de 1 (=nada de acuerdo) a 5 (=totalmente de acuerdo).

\begin{tabular}{|c|c|c|c|}
\hline & $\begin{array}{l}\text { Nada de } \\
\text { acuerdo }\end{array}$ & $\leftrightarrow$ & $\begin{array}{l}\text { Totalmente } \\
\text { de acuerdo }\end{array}$ \\
\hline $\begin{array}{l}\text { La Dirección General } \\
\text { se preocupa por seguir } \\
\text { directamente los temas de } \\
\text { innovación de la empresa } \\
\text { (clientes, proveedores, } \\
\text { socios, etc.) }\end{array}$ & & & \\
\hline $\begin{array}{l}\text { La innovación forma parte } \\
\text { de la estrategia básica de la } \\
\text { empresa }\end{array}$ & & & \\
\hline $\begin{array}{l}\text { En el futuro próximo, la } \\
\text { cooperación para innovar } \\
\text { será un factor estratégico }\end{array}$ & & & \\
\hline $\begin{array}{l}\text { Existe una preocupación } \\
\text { constante por detectar } \\
\text { las innovaciones en la } \\
\text { competencia }\end{array}$ & & & \\
\hline $\begin{array}{l}\text { Existe una actitud constante } \\
\text { por incrementar las } \\
\text { inversiones en nuevas } \\
\text { tecnologías }\end{array}$ & & & \\
\hline $\begin{array}{l}\text { Su empresa busca } \\
\text { constantemente información } \\
\text { sobre las nuevas tecnologías } \\
\text { que pueda aplicar }\end{array}$ & & & \\
\hline $\begin{array}{l}\text { La inversión en innovación } \\
\text { seguro que genera una } \\
\text { mayor rentabilidad futura }\end{array}$ & & & \\
\hline $\begin{array}{l}\text { La dirección de la empresa } \\
\text { se preocupa de que los } \\
\text { técnicos de la empresa } \\
\text { tengan actualizados sus } \\
\text { conocimientos }\end{array}$ & & & \\
\hline $\begin{array}{l}\text { Existe un inventario } \\
\text { actualizado de la dotación } \\
\text { tecnológica de la empresa }\end{array}$ & & & \\
\hline $\begin{array}{l}\text { La empresa se adapta a los } \\
\text { cambios del entorno rápida y } \\
\text { adecuadamente }\end{array}$ & & & \\
\hline $\begin{array}{l}\text { Se incorporan habitualmente } \\
\text { mejoras en los productos, } \\
\text { procesos y sistemas }\end{array}$ & & & \\
\hline $\begin{array}{l}\text { En todos los ámbitos existe } \\
\text { una preocupación continua } \\
\text { por la calidad del producto o } \\
\text { servicio }\end{array}$ & & & \\
\hline $\begin{array}{l}\text { Existe una cultura de } \\
\text { acumular y transmitir las } \\
\text { experiencias a los nuevos } \\
\text { trabajadores }\end{array}$ & & & \\
\hline $\begin{array}{l}\text { Se potencia la creatividad de } \\
\text { los trabajadores }\end{array}$ & & & \\
\hline
\end{tabular}

6. Indique los siguientes resultados relacionados con la innovación de modo aproximado de los últimos tres años.

\begin{tabular}{|l|l|l|l|}
\hline \multirow{2}{*}{ ASPECTOS } & \multicolumn{3}{c|}{$\begin{array}{c}\text { RESULTADOS } \\
\text { (ÚLTIMOS 3 AÑOS) }\end{array}$} \\
\cline { 2 - 4 } & 2000 & 2001 & 2002 \\
\hline Número de nuevos productos de éxito & & & \\
\hline Número de nuevos procesos de éxito & & & \\
\hline Cuota de mercado de nuevos productos & & & \\
\hline Número de mejoras de productos existentes & & & \\
\hline Número de mejoras de procesos existentes & & & \\
\hline $\begin{array}{l}\text { Tasa de renovación de productos (Nuevos o } \\
\text { mejorados) }\end{array}$ & & & \\
\hline $\begin{array}{l}\text { Tasa de renovación de procesos (Nuevos o } \\
\text { mejorados) }\end{array}$ & & & \\
\hline Número de patentes y modelos de utilidad & & & \\
\hline Número de marcas registradas & & & \\
\hline Número de licencias & & & \\
\hline $\begin{array}{l}\text { Porcentaje de volumen de negocio debido a } \\
\text { las innovaciones respecto al total de ventas }\end{array}$ & & & \\
\hline
\end{tabular}

7 En una escala de 1 (=nada satisfecho) a 5 (=muy satisfecho) evalúe el grado de satisfacción con los resultados de innovación obtenidos en su empresa en los últimos 3 años.

\begin{tabular}{|c|c|c|c|c|}
\hline \multicolumn{5}{|c|}{ SATISFACCIÓN CON LOS } \\
\hline $\begin{array}{c}\text { Nada } \\
\text { satisfecho }\end{array}$ & $\begin{array}{c}\text { Algo } \\
\text { satisfecho }\end{array}$ & $\begin{array}{c}\text { Satisfacción } \\
\text { normal }\end{array}$ & $\begin{array}{c}\text { Bastante } \\
\text { satisfecho }\end{array}$ & $\begin{array}{c}\text { Muy } \\
\text { satisfecho }\end{array}$ \\
\hline & & & & \\
\hline
\end{tabular}

8. Señale si alguno de los siguientes departamentos/servicios/secciones de su empresa está orientado específicamente a la innovación (nota: en la casilla se incluye la codificación interna de valores otorgados a esta pregunta).

\begin{tabular}{|c|l|}
\hline $\begin{array}{c}\text { Marque si } \\
\text { existe }\end{array}$ & \multicolumn{1}{c|}{ DEPARTAMENTO/SERVICIO/SECCIÓN } \\
\hline 5 & Innovación \\
\hline 5 & I+D \\
\hline 4 & Diseño \\
\hline 3 & Calidad \\
\hline 2 & Métodos y procedimientos \\
\hline 1 & Gestión medioambiental \\
\hline 1 & Otros \\
\hline
\end{tabular}

9 Indique la actividad o actividades que realiza su empresa:

10. Señale su facturación anual aproximada en $€$, al igual que la plantilla media anual de empleados.

\begin{tabular}{|c|l|c|}
\hline $\begin{array}{c}\text { Facturación de la empresa } \\
\text { en el año } 2002 \text { (en } € \text { ) }\end{array}$ & & $\begin{array}{c}\text { Número medio de empleados } \\
\text { en el año } 2002\end{array}$ \\
\hline & & \\
\hline
\end{tabular}


CARLOS M. FERNÁNDEZ-JARDÓN FERNÁNDEZ es profesor titular de Economía Aplicada en la Universidad de Vigo. Licenciado en Matemáticas, MBA por la Escuela de Negocios Caixavigo, es doctor en Ciencias y posteriormente obtuvo un segundo doctorado en Economía por la U. de Navarra. Sus líneas de investigación se centran en la intersección de capital intelectual, gestión del conocimiento, internacionalización de Pymes, y clúster de empresas.

MIGUEL GONZÁLEZ-LOUREIRO es profesor de Dirección de Empresas en la Universidad de Vigo. Licenciado en Ciencias Económicas y Empresaria- les, es doctor en Dirección de Empresas por la U. de Vigo. Sus ámbitos de investigación se sitúan en la intersección del proceso de internacionalización, gestión de intangibles, clúster de empresas y el área de aspectos cognitivos de la estrategia.

JOSÉ PITA CASTELO es profesor de Marketing en la Universidad de Vigo. Licenciado en psicología, es Doctor en Ciencias Económicas y Empresariales por la Universidad Complutense de Madrid. Sus líneas de investigación se centran en temas de marketing e investigación de mercados relacionados con servicios e innovación. 